ORIGINAL STUDY

\title{
A safe way to find the posterior ethmoidal cells: navigation with cottonoid
}

\author{
Carlos Miguel Chiesa Estomba, Frank Alberto Betances Reinoso, Carmelo Santidrian Hidalgo \\ Otorhinolaryngology - Head \& Neck Surgery Department, University Hospital of Vigo, Spain
}

\begin{abstract}
BACKGROUND. Functional endoscopic sinus surgery (FESS) is a reliable option in the treatment of sinus pathology, but the presence of the anatomical variant and difficult cases like massive polyposis or revision FESS can generate some problems to surgeons.

MATERIAL AND METHODS. After performing an unciformectomy, a partial anterior ethmoidectomy and maxillary ostium antrostomy, we slide a cottonoid back to the basal lamella of the middle turbinate with a Cottle dissector and introduce it in the superior meatus. After that, we return to the middle meatus and proceed to open the basal lamella finding the cottonoid placed there previously.

RESULTS. An easy technique, safe and reproducible, that allows us to advance in our dissection, avoiding damaging important structures.

CONCLUSION. In this paper we present a safe way to approach the posterior ethmoidal cells complex in the classic way through the basal lamella of the middle turbinate, under the guidance of a cottonoid, a safe and easy maneuver to do this procedure in the beginning of our formation or in complex cases.
\end{abstract}

KEYWORDS: posterior ethmoid cells, FESS, cottonoid

\section{INTRODUCTION}

Surgical intervention in the treatment of sinus disease is considered in patients who fail to improve after a trial of maximal medical treatment. Functional endoscopic sinus surgery involves the clearance of polyps and polypoid mucosa and opening the sinus ostium. Removal of inflammatory tissue and reduction of the load of antigens inciting that inflammation, as well as the improvement of sinus ventilation and mucociliary clearance, are the probable mechanisms, whereby functional endoscopic sinus surgery (FESS) improves symptoms in nasal polyposis ${ }^{1}$.

Endoscopic sinus surgery is a technically demanding procedure, requiring strong endoscopy skills ${ }^{2}$. However, FESS performed with a meticulous technique significantly decreased patient morbidity and trauma ${ }^{3}$.

Commonly, endoscopic sinus surgery training has relied on cadaveric dissections ${ }^{4}$ and more recently the virtual reality simulation (Endoscopic Sinus Surgery Simulator ES3) has proven to be a valuable and effective method allowing surgeons to prepare for sinus surgery $^{5}$. But, the anatomical variant and difficult cases like massive polyposis or revision FESS can generate some problems in the beginning of our training and in more experienced surgeons. Therefore, the perfect description of different landmarks and their relations with the most important structures in the vicinity of the nasal corridor may decrease this risk.

Alternatives like navigation appear as an option to try to decrease this risk, but this technology is not available in all the centers. For this reason, in this paper we present a safe step to approach the posterior ethmoidal cells complex in the classic way through the basal lamella of the middle turbinate, under the guidance of a cottonoid, a safe and easy manoeuvre to do this procedure in the beginning of our formation or in complex cases. 


\section{MATERIAL AND METHODS}

Based on the 4-lamellae technique described by Messerklinger and popularized by Stammberger ${ }^{3}$, we describe below a simple way to be sure when we cross the basal lamella of the middle turbinate to access the posterior ethmoid. We use this technique in our center when the resident starts this type of surgery or in cases of massive polyposis or revision FESS.

We start our procedure doing an unciformectomy, after that we perform a partial anterior ethmoidectomy, expanding the ostium of the maxillary sinus. Once we control all the landmarks, we can see the basal lamella of the middle turbinate in the middle meatus. After that, we let slip a cottonoid back to the basal lamella of the middle turbinate with a Cottle dissector and introduce it in the superior meatus. In some cases, we can find polyps in the superior meatus, and the cottonoid can be a bit more complicated to introduce, but not impossible (Figure 1).

After that, we return to the middle meatus and proceed to open the basal lamella, maintaining a bridge into the lower level of at least 1-2 $\mathrm{mm}$, to avoid destabilizing the turbinate. The first structure that we will find will be the cottonoid placed where we previously left it (Figure 2).

We can repeat this manoeuvre in the contralateral middle meatus, doing our dissection safer and being possible to be sure when we address the posterior ethmoid.

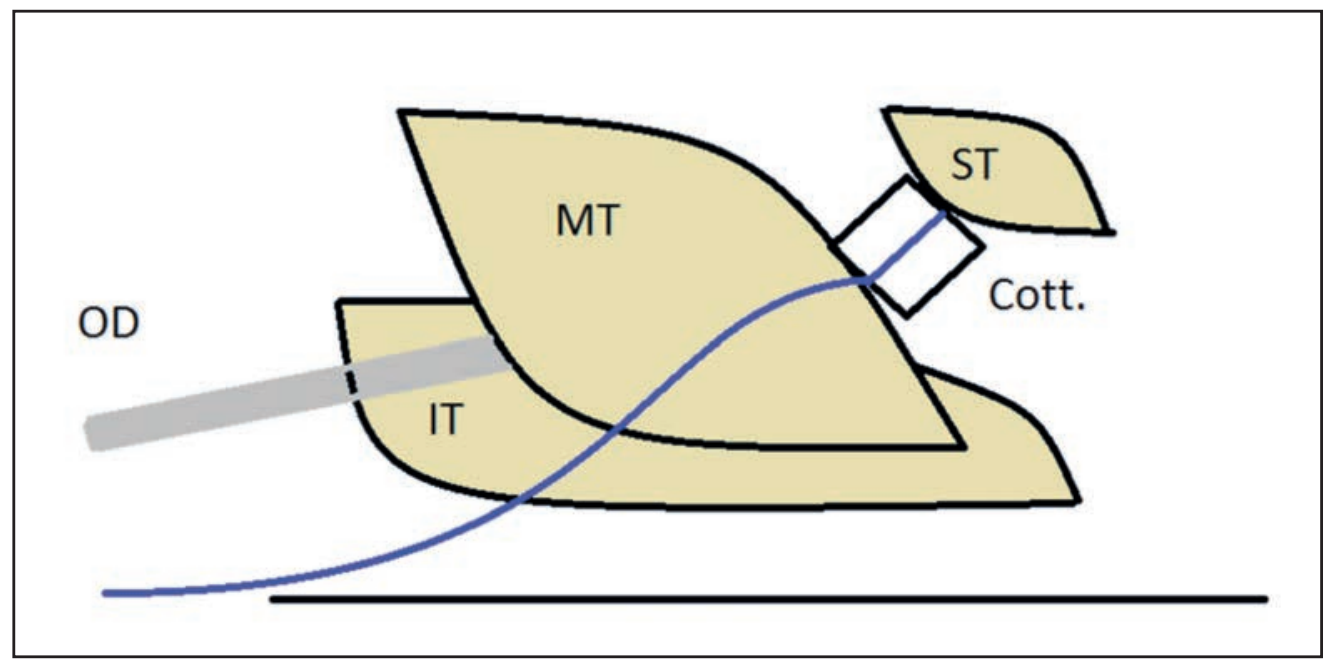

Figure 1 Schematic representations of cottonoid introduction into the superior meatus, between the middle and superior turbinate (ST: superior turbinate, MT: middle turbinate, IT: inferior turbinate, Cott: cottonoid, OD: optical device)

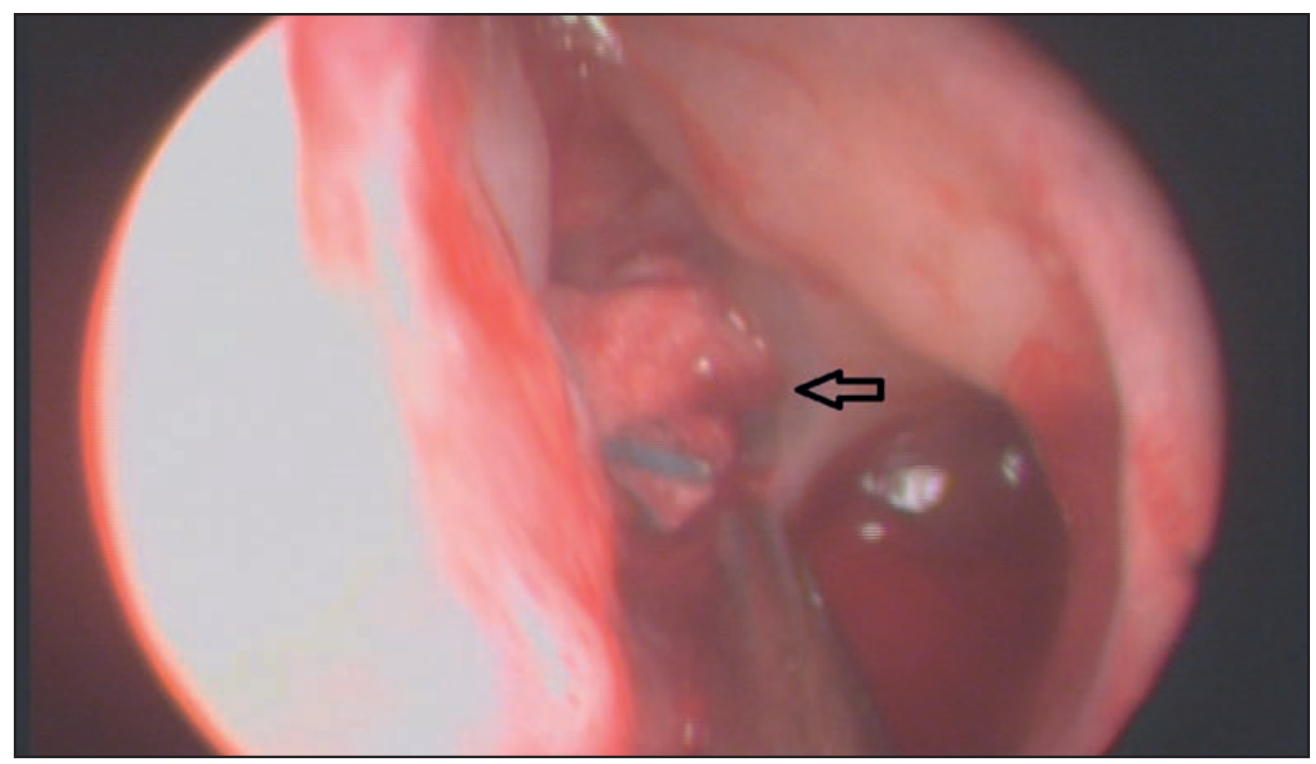

Figure 2 Vision of the left middle meatus, after opening the basal lamella; one can see the cottonoid indicated by the black arrow 


\section{DISCUSSIONS}

The basal lamella of the middle turbinate is the third basal lamella of the ethmoturbinals ${ }^{6}$. The basal lamella of the middle turbinate separates the anterior ethmoid from the posterior ethmoid. This structure has been known before as "ground" lamella, a term that has been abandoned today in surgical anatomy ${ }^{7}$.

This represents an important landmark in FESS due to its location in the middle of the ethmoidal labyrinth and the important relation with other vital structures, therefore it is important to know that surgical misadventures can seriously damage vital structures that surround this area, including the skull base, brain, eyes or vessels. For that reason, it is not surprising that sinus surgery has become the most common cause for litigation in the field of otolaryngology ${ }^{8}$.

In this paper we propose a simple way to access the posterior ethmoid through the basal lamella of the middle turbinate, under the guidance of a cottonoid that will allow us to certify our location before moving forward in our dissection. This technique will be especially useful when performing FESS in patients with massive polyposis or in case of revision surgery, but also to those residents who are initiated in the FESS technique.

\section{CONCLUSIONS}

FESS is a reliable option in the treatment of sinus pathology, but is necessary to know in detail the anatomical landmarks to try to avoid injuring the important anatomical structures that are closely related to the nasal corridor. One of the key and most difficult steps to perform in this technique is usually to pass through the basal lamella to the posterior ethmoid cells. It is why we propose the use of a cottonoid to serve as reference, which will allow us to certify our location before moving forward with our dissection.

Conflict of interest: The authors have no conflict of interest.

Contribution of authors: All authors have equally contributed to this work.

\section{REFERENCES}

1. Fokkens W.J., Lund V.J., Mullol J., Bachert C., Alobid I., Baroody F., et al. - European Position Paper on Rhinosinusitis and Nasal Polyps 2012. Rhinol Suppl., 2012;(23):3:1-298.

2. Kennedy D.W. - Functional endoscopic sinus surgery: technique. Arch Otolaryngol Head Neck Surg., 1985;111:643-649.

3. Stammberger H., Posawetz W. - Functional endoscopic sinus surgery. Eur Arch Otorhinolaryngol., 1990;247:63-76.

4. Malekzadeh S., Pfisterer M.J., Wilson B., Na H., Steehler M.K. - A novel low-cost sinus surgery task trainer. Otolaryngol Head Neck Surg., 2011;145:530-533.

5. Fried M.P., Sadoughi B., Gibber M.J., et al. - From virtual reality to the operating room: the endoscopic sinus surgery simulator experiment. Otolaryngol Head Neck Surg., 2010;142:202-207.

6. Simmen D., Jones N. - Manual of Endoscopic Sinus and Skull Base Surgery. Thieme, 2014.

7. Lund V.J., Stammberger H., Fokkens W.J., Beale T., Bernal-Sprekelsen M., Eloy P., et al. - European position paper on the anatomical terminology of the internal nose and paranasal sinuses. Rhinol Suppl., 2014;(24):134.

8. Pelausa E.O., Smith K., Dempsey I. - Orbital complications of functional endoscopic sinus surgery. J Otolaryngol., 1995;24:154-159. 